\title{
Quantum interaction among intense laser beams in vacuum
}

\author{
A. Di Piazza ${ }^{a}$ and K.Z. Hatsagortsyan ${ }^{b}$ \\ Max-Planck-Institut für Kernphysik, Saupfercheckweg 1, 69117 Heidelberg, Germany
}

\begin{abstract}
Quantum electrodynamics (QED) predicts that electromagnetic fields interact among each other also in vacuum. We study the possibility of experimentally revealing this interaction by using soon available laser fields with intensities of order of $10^{23}-10^{26} \mathrm{~W} / \mathrm{cm}^{2}$. First, a few processes are first reviewed where vacuum polarization effects can be detected, like laser-assisted photon-photon scattering and the light diffraction by a strong standing wave. The possibility of enhancing these effects by using a plasma is also mentioned. Finally, the process of photon splitting in a laser field is discussed in detail together with its possible experimental observation.
\end{abstract}

\section{Introduction}

QED is the best-tested physical theory we have. The predictions of QED especially in atomic and particle physics have been confirmed by experiments with very high accuracy, like those on the anomalous magnetic moment of the electron or the Lamb shift. However, most of these tests relate to perturbative QED. This means that, from a theoretical point of view, the theoretical predictions are based on the Dyson expansion of the S-matrix in the fine structure constant $\alpha=e^{2} / 4 \pi \approx 1 / 137$ up to the required accuracy (here $-e<0$ is the electron charge, natural units with $\hbar=c=1$ are used) [1]. On the other hand, testing QED in the presence of strong classical electromagnetic fields so far has only been successful in the case of strong Coulomb fields, i.e. the fields created by highly-charged nuclei with charge number $Z \lesssim 1 / \alpha \approx 137$. The Coulomb field of a nucleus with $Z \sim 1 / \alpha$ is, at the typical QED distance $\lambda_{c}=1 / \mathrm{m}$ (Compton length) with $m$ being the electron mass, comparable to the so-called critical electric field $E_{c r}=m^{2} / e=1.3 \times 10^{16} \mathrm{~V} / \mathrm{cm}$, i.e. it is able to produce electron-positron pairs in vacuum [2]. The Coulomb field produced by highly-charged nuclei is so strong that it has to be exactly taken into account in the calculation of the amplitudes of different QED processes [1]. In other words, expanding the amplitude of a process with respect to the nuclear field (or, more precisely, with respect to the parameter $Z \alpha$ ) and keeping only a few terms in the expansions leads to inaccurate results; nonperturbative effects become important and cannot be neglected. Vacuum polarization effects in strong Coulomb fields like the Delbrück scattering, i.e. the scattering of a photon by the Coulomb field of a heavy nucleus, or photon splitting in a strong Coulomb field have been predicted and studied theoretically [3,4] and confirmed experimentally [5,6].

Other classes of strong fields that have been considered in the literature are static and uniform magnetic fields. In general, from a theoretical point of view, the consistent inclusion of a strong magnetic field in the calculation of QED cross-sections is easier than including nuclear fields. In fact, in the former case the vacuum is stable: spontaneous electron-positron pair creation from vacuum is forbidden by energy conservation [7]. The typical strength of a magnetic

\footnotetext{
a e-mail: dipiazza@mpi-k.de

b e-mail: k.hatsagortsyan@mpi-k.de
} 
field in which nonperturbative QED effects become apparent is given by the so-called critical magnetic field $B_{c r}=m^{2} / e=4.41 \times 10^{13} \mathrm{G}$ (see the books [8,9] for a recent review). Due to the extremely large value of $B_{c r}$, nonperturbative QED effects in the presence of strong magnetic fields can significantly occur only in astrophysical environments like around highly magnetized neutron stars [10-12].

Nowadays, strong lasers are opening up important perspectives in different fields. For example, available table-top multiterawatt lasers are employed to create new x-ray and even $\gamma$-ray radiation sources [13] as well as to accelerate electrons, protons and ions to high energies [14]. Moreover, theoretical proposals have been already put forward to reach the critical electric field value $E_{c r}=1.3 \times 10^{16} \mathrm{~V} / \mathrm{cm}$ by focusing the high-order harmonics generated in the reflection of a strong laser beam by a plasma surface [15]. Laser fields have clearly a very different nature with respect to the static and uniform magnetic fields and to the Coulomb fields. For this reason they provide a unique tool for testing QED in the nonperturbative regime in the presence of space-time-dependent fields. In the present paper we will mainly consider processes in which the laser field intensity is much lower than the critical intensity $I_{c r}=E_{c r}^{2} / 8 \pi=2.3 \times 10^{29} \mathrm{~W} / \mathrm{cm}^{2}$. In fact, the strongest laser field ever produced in a laboratory has an intensity of "only" $7 \times 10^{21} \mathrm{~W} / \mathrm{cm}^{2}$ and has been obtained by focusing a $45-\mathrm{TW}$ Ti-Sapphire laser beam onto a volume with a typical length of the order of the laser wavelength $\lambda=800 \mathrm{~nm}$ [16]. In general, below the critical field, spontaneous pair creation in vacuum is exponentially suppressed and completely negligible [17]. However, vacuum manifests nonlinear properties due to the continuous creation and annihilation of virtual electron-positron pairs. Photon-photon scattering [18] represents the vacuum nonlinearity at lowest order in the field of two photons. The nonlinear electromagnetic properties of quantum vacuum in the presence of strong laser fields are attracting the interest of a large physical community. Numerous recent publications are devoted to the possibility of experimentally detecting some effects of vacuum nonlinearity [19-26] (see also the recent reviews [27-29]). So far, the only successful experimental test of vacuum nonlinearity has been achieved in the production of electron-positron pairs in the collision of a high-energy electron beam with a strong laser pulse [30].

In the following we will first mention the possibility that due to the vacuum nonlinearity, harmonics of the lasers are created in vacuum in the collision of two equal strong laser beams: the photon-photon scattering would be the lowest order process in which two laser photons interact and only change their propagation directions. Then, we will also mention how important it is to take into account the focusing of strong laser fields when describing their interaction in vacuum (as we will see, this interaction is qualitatively similar to the standard light diffraction by an aperture). Moreover, the possibility of enhancing the effects of vacuum polarization by employing a cold high-density plasma is shortly reviewed. Finally, the process of photon splitting in a laser field is discussed in more detail. Photon splitting cannot occur in vacuum due to the fact that QED is charge-parity invariant [1]. However, as we have already mentioned, if an external field, e.g. a Coulomb or a magnetic field, is present this process is allowed. Apart from the above mentioned calculations of photon splitting in the presence of a Coulomb field and of a magnetic field, the photon splitting process has also been considered in the presence of a crossed field (a constant electromagnetic field with the electric and magnetic fields having the same strength and being perpendicular to each other) [31]. This case is relevant because a strong, low-frequency laser field (more precisely such that the parameter $\xi=e E / m \omega$ is very large, with $E$ and $\omega$ being the laser amplitude and frequency, respectively) can be approximated by a crossed field. It is interesting to observe that the kinematics of photon splitting depends essentially on the structure of the external field. The most complicated situation occurs in the presence of a Coulomb field, where the wave vector $\boldsymbol{k}_{1}$ of the initial photon and the wave vectors $\boldsymbol{k}_{2}$ and $\boldsymbol{k}_{3}$ of the final ones are, in general, not coplanar. In a constant and uniform electromagnetic field, all the initial and final photons propagate almost in the same direction (up to nonzero photon mass due to the polarization of vacuum in the field). In a laser plane wave the initial and final photons are coplanar in the frame where the laser wave and the initial photons are counterpropagating. These features can be easily deduced from energy-momentum conservation. 
The paper is organized as follows. In the next section, vacuum-harmonic generation, light diffraction by a strong standing wave and the enhancement of vacuum polarization effects in a plasma are shortly reviewed. In section 3 , the photon splitting process in a linearly polarized monochromatic laser field is discussed and finally, in section 4, the main conclusions are drawn.

\section{Vacuum polarization effects in strong laser beams}

As we have mentioned, we will consider here a few processes arising from the nonlinearity of vacuum in the presence of a strong electromagnetic wave.

\subsection{Vacuum high-order harmonic generation}

Atomic high-order harmonic generation is a well-known process. Its general features can be qualitatively understood by means of the so-called three-step model: the bound electron in the presence of the laser field tunnels to the continuum, moves freely in the laser field and then recombines with the parent ion, emitting radiation [32]. Something similar can happen in vacuum when causing two strong laser beams to collide [23]. In fact, the virtual electronpositron pairs present in the vacuum can absorb a large number of laser photons and then annihilate and emit only a few high-energy photons. In the situation we have considered in [23], two equal laser beams are caused to collide head on and, in this case, it is possible to show that odd and even harmonics of the laser are produced in vacuum. In order to prime the nonperturbative process of high-order harmonic generation in vacuum, lasers of an intensity comparable to $I_{c r}$ are required. In this case, it can be shown that a wide plateau of harmonics is produced with a cut-off approximately at $\sqrt{e E}$, with $E$ being the laser amplitude. At laser intensities much lower than $I_{c r}$ the production of high harmonics is strongly suppressed. In this case, that corresponds to the actual experimental situation, only the production of the first harmonic is observable. This process corresponds to the scattering of two laser photons that only change their propagation direction. In order to significantly increase the number of photons scattered, a third "assisting" laser can be shown in the collision region. As a result, it has been shown that the "laser-assisted" photon-photon scattering can be observed in principle by using present-day lasers with a power of the order of $100 \mathrm{TW}$ [23].

\subsection{Light diffraction by a strong standing wave}

It is not surprising that, due to the polarization of vacuum, the presence of a strong laser beam in vacuum modifies the propagation of a probe beam that passes through it. In the description of this kind of interaction, the so-called refractive-index approach is usually employed, i.e. the region of space where the strong field is present is described as a dielectric material with refractive indices different from unity. The refractive indices can be calculated according to QED and depend on the mutual polarization of the strong and the probe beam [9]. However, the concept of refractive index is a concept valid for macroscopic interaction lengths $l$ such that $l^{2} \gg \lambda d$ with $\lambda$ being the wave wavelength and $d$ the distance between the interaction region and the detector. Now, in order to obtain a strong field with an intensity such that QED processes become manifest, it is necessary to focus the laser beam into a microscopic region with a linear extension of the order of $1 \mu \mathrm{m}$ (it is understood that an optical field is used to polarize the vacuum). We have shown in [25] that when a probe electromagnetic beam passes through a strong laser beam, the refractive-index approach breaks down. In fact, the probe beam is "diffracted" by the strong laser beam so that it is not possible to describe the strong laser as a dielectric medium. The theoretical predictions of the two approaches are different both qualitatively and quantitatively. For instance, the refractive-index approach predicts that after passing through an optical standing wave, a linearly polarized x-ray probe beam should become elliptically polarized and the main axis of the ellipse lies along the initial probe polarization direction. The diffraction theory predicts that in addition, the main axis 
of the ellipse is rotated with respect to the initial probe polarization [25]. The quantitative predictions of the two theories are very different as well. In fact, the approximated refractiveindex approach overestimates by more than one order of magnitude the effects on the ellipticity acquired by the probe after the interaction. As the the experimental detection of this process of light-by-light diffraction, we have estimated the effect by employing the following parameters. We have considered an optical laser beam with parameters available in the near future at the Extreme Light Infrastructure (ELI): $\omega=1 \mathrm{eV}$ and intensity $I \approx 10^{25}-10^{26} \mathrm{~W} / \mathrm{cm}^{2}$ [33]. The wavelength of the $\mathrm{x}$-ray probe beam is set to $0.4 \mathrm{~nm}$. Finally, by assuming the strong field to be focused to the diffraction limit and by using the diffraction theory we obtain an ellipticity and a polarization rotation angle of the order of $10^{-6}-10^{-5}$ rad. Those very small values are already measurable nowadays in the x-ray regime [34] even though this proves a very challenging experiment. More details about the experimental aspects of this process can be found in [25].

\subsection{Enhancement of vacuum polarization effects in a plasma}

It is clear from the foregoing discussion that in order to observe vacuum polarization effects, very intense laser fields are required. Is there a way to enhance these effects at a fixed laser intensity? We have found a positive answer to this question by considering the propagation of a strong laser field through a cold plasma. Since the wave intensity is supposed to be about $10^{22}-10^{23} \mathrm{~W} / \mathrm{cm}^{2}$, the plasma is highly relativistic and completely ionized. If we assume for simplicity that the plasma is homogeneous and that a two-fluid (electron and ion fluids) description of the plasma can be used (cold plasma approximation), it is then possible to show that, by accounting for vacuum polarization, in the presence of a circularly polarized plane wave the plasma refractive index is determined by the formula [35]

$$
n_{0}=\sqrt{n_{0, p l}^{2}+\frac{2 \alpha}{45 \pi} \frac{E^{2}}{E_{c r}^{2}}\left(1-n_{0, p l}^{2}\right)^{2}} .
$$

In this expression,

$$
n_{0, p l}=\sqrt{1-\frac{1}{\omega^{2}}\left(\frac{\omega_{p l, e}^{2}}{\gamma_{e}}+\frac{\omega_{p l, i}^{2}}{\gamma_{i}}\right)}
$$

is the plasma relativistic refractive index experienced by the circularly polarized wave in the absence of vacuum polarization effects [36], with $\omega_{p l, e / i}$ being the plasma frequencies and $\gamma_{e / i}$ the relativistic Lorentz factors of the electrons and the ions in the plasma (due to the high laser intensities the motion of the ions has to be taken into account, too).

From another side, if we apply the refractive index approach in vacuum in the presence of a strong electric field with amplitude $E$, the typical expression of the vacuum refractive index then is $[9]$

$$
n=1+\frac{\alpha}{45 \pi} \frac{E^{2}}{E_{c r}^{2}} .
$$

Thus, it is evident that the presence of the plasma allows for considering situations in which $n_{0, p l} \ll 1$ and the refractive index of the plasma would scale as the square root of the vacuum correction. By exploiting the peculiar behaviour of the plasma in the presence of a wave with a frequency near the plasma frequency, i.e. at $n_{0, p l} \ll 1$, an enhancement of vacuum polarization effects of more than one order of magnitude has been predicted theoretically in [35]. In the same paper other aspects of the problem are addressed, like the presence of collisions between electrons and ions which can be determinant in the regime of laser frequencies slightly above the plasma frequency. Experimental issues like plasma stability and other related processes are discussed as well. 


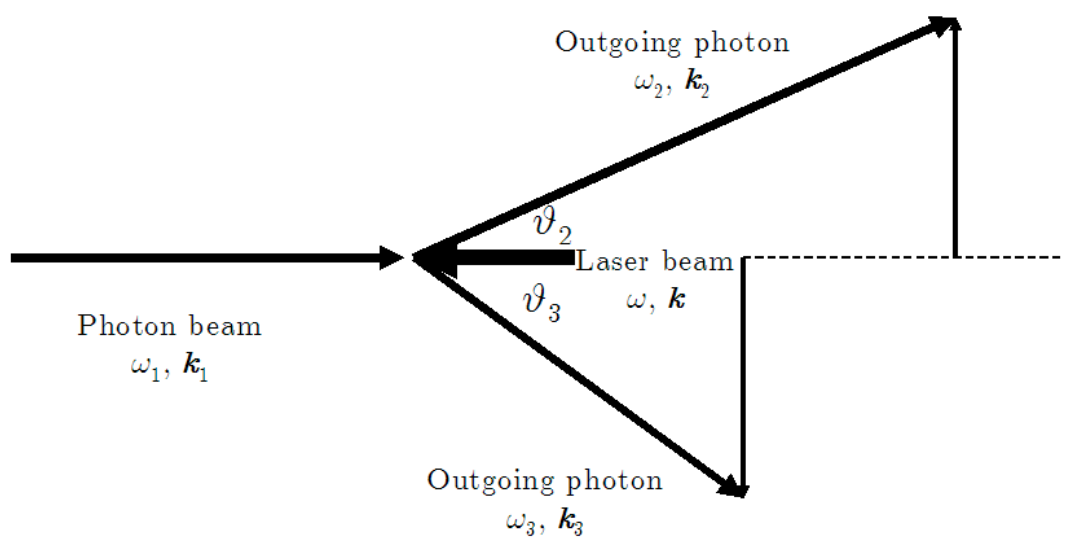

Fig. 1. The geometry of the collision of a photon beam with energy $\omega_{1}$ with a strong laser field whose photons have four-momentum $(\omega, \boldsymbol{k})$. Due to energy-momentum conservation all the initial and final photons propagate in the same plane. The outgoing photons resulting from the splitting can be characterized by their energies $\omega_{2}$ and $\omega_{3}$ and by their propagation angles $\vartheta_{2}$ and $\vartheta_{3}$, respectively.

\section{Photon splitting in a laser field}

In this section we study the QED process of photon splitting in a laser field [37]. The geometry of the collision is sketched in Fig. 1. We assume without loss of generality that the initial photon and the laser beam are counterpropagating along, say, the $z$ axis. Due to energy-momentum conservation, the two outgoing photons lie in a plane together with the laser propagation direction and the initial photon direction. As a general consideration, we expect that the amplitude of the photon splitting process depends only on the two gauge- and Lorentz-invariant parameters

$$
\kappa=\frac{\omega_{1} \omega}{m^{2}}, \quad \chi=\frac{\omega_{1}}{m} \frac{E}{E_{c r}},
$$

where $\omega_{1}$ is the energy of the initial photon. The parameter $\kappa$ is half of the square of the total energy of the initial photon and of a laser photon in their center-of-momentum system. Instead, the parameter $\chi$ is the so-called quantum intensity parameter responsible for the magnitude of the nonperturbative effects induced by the strong laser field.

The amplitude $\mathcal{M}$ of the photon-splitting process of a real photon with four-momentum $k_{1}^{\mu}=\left(\omega_{1}, \boldsymbol{k}_{1}\right)$ into two real photons with four-momenta $k_{2}^{\mu}=\left(\omega_{2}, \boldsymbol{k}_{2}\right)$ and $k_{3}^{\mu}=\left(\omega_{3}, \boldsymbol{k}_{3}\right)$ is given by

$$
\mathcal{M}=i e^{3} \int d^{4} x \operatorname{Tr}\left\langle x\left|\mathrm{e}^{-i\left(k_{1} x\right)} \hat{e}_{1} \mathcal{G}[\mathcal{A}] \mathrm{e}^{i\left(k_{2} x\right)} \hat{e}_{2}^{*} \mathcal{G}[\mathcal{A}] \mathrm{e}^{i\left(k_{3} x\right)} \hat{e}_{3}^{*} \mathcal{G}[\mathcal{A}]\right| x\right\rangle+\left(k_{2} \leftrightarrow k_{3}, e_{2} \leftrightarrow e_{3}\right),
$$

where $e_{i}^{\mu}$ with $i=1,2,3$ are the polarization four-vectors of the initial and final photons and where

$$
\mathcal{G}[A]=\frac{1}{\gamma^{\mu}\left(\mathrm{i} \partial_{\mu}+e A_{\mu}(x)\right)-m+\mathrm{i} 0}
$$

is the dressed electron propagator in a laser field with four-potential $A^{\mu}(x)$ [37]. In order to include the presence of the strong laser we have used the so-called Furry picture in which the laser field is exactly included in the free Hamiltonian of the electron-positron field, giving rise to the Volkov states as one-particle electron and positron states [1]. The explicit evaluation of the amplitude (5) is cumbersome but it can be performed by employing the operator technique developed in $[38,39]$. The details of this calculations can be found in [37] where the expression of the amplitude has been calculated for any plane wave arbitrarily polarized. Below, we give the expressions of the different amplitudes in the simpler case of a linearly polarized, monochromatic laser field and in the parameter region where $\xi=\chi / \kappa \gg 1$, with $\chi$ being fixed (this implies that 
the parameter $\kappa$ is assumed to be much smaller than unity). This case corresponds physically, for example, to a strong optical laser field like that expected at the ELI facility [33]. It is interesting to observe that if the laser field were circularly polarized then, due to the conservation of the component of the total angular momentum along the propagation direction and to the fact that all the laser photons have the same elicity, only one or three photons could be absorbed from the laser [37]. This is true in the parameter region we are considering here because the energy of the laser photons is very small (more precisely, $\kappa \ll 1$ ) and so that the final photons are almost collinear to the initial high-energy photons. If the laser field is linearly polarized, then the total (laser field plus photon field) angular momentum is again conserved, but the laser photons have no definite elicity and any number of laser photons can be absorbed in this case. However, we stress that due to the Furry theorem only an odd number of laser photons can be absorbed [1]. We indicate as $M_{n, i j k}$ the amplitude corresponding to the photon splitting of a photon with polarization $i$ into two photons with polarizations $j$ and $k$ respectively with $i, j, k \in\{1,2\}$ and with absorption of $n$ laser photons. It can be shown that at leading order in $\kappa$ the only amplitudes different from zero are:

$$
\begin{aligned}
& M_{n, 111}=\frac{i^{\frac{n+3}{2}} \sqrt{2} m e^{3} \chi^{3}}{\pi^{2}} \iiint_{0}^{\infty} d s_{1} d s_{2} d s_{3} \mathrm{e}^{-i\left(S+\psi_{0}\right)}\left\{-\frac{i\left(z_{1}+z_{2}+z_{3}\right) \mathcal{D}_{n}^{(1)}\left(\psi_{0}\right)}{2 S^{2} \chi^{2}}\right. \\
& +S \mathcal{D}_{n}^{(2)}\left(\psi_{0}\right)\left[2 z_{1} z_{2} z_{3}-\nu_{2} \nu_{3}\left(1+t_{1}^{2}\right) z_{1}+\nu_{2}\left(1+t_{2}^{2}\right) z_{3}+\nu_{3}\left(1+t_{3}^{2}\right) z_{2}\right. \\
& \left.\left.-4 \nu_{2} \nu_{3}+\nu_{2} \nu_{3}\left(1+t_{1}\right)\left(1+t_{2}\right)\left(1+t_{3}\right)\right]\right\}+\widetilde{\mathcal{G}}_{n}(1)+\widetilde{\mathcal{G}}_{n}\left(-\nu_{2}\right)+\widetilde{\mathcal{G}}_{n}\left(-\nu_{3}\right) ; \\
& M_{n, 122}=\frac{i^{\frac{n+3}{2}} \sqrt{2} m e^{3} \chi^{3}}{\pi^{2}} \iiint_{0}^{\infty} d s_{1} d s_{2} d s_{3} \mathrm{e}^{-i\left(S+\psi_{0}\right)}\left\{-\frac{i z_{1} \mathcal{D}_{n}^{(1)}\left(\psi_{0}\right)}{2 S^{2} \chi^{2}}\right. \\
& \left.-S \mathcal{D}_{n}^{(2)}\left(\psi_{0}\right) \nu_{2} \nu_{3}\left(1-t_{1}\right)\left[\left(1+t_{1}\right) z_{1}+1-t_{2} t_{3}\right]\right\}+\widetilde{\mathcal{G}}_{n}(1) ; \\
& M_{n, 212}=\frac{i^{\frac{n+3}{2}} \sqrt{2} m e^{3} \chi^{3}}{\pi^{2}} \iiint_{0}^{\infty} d s_{1} d s_{2} d s_{3} \mathrm{e}^{-i\left(S+\psi_{0}\right)}\left\{-\frac{i z_{2} \mathcal{D}_{n}^{(1)}\left(\psi_{0}\right)}{2 S^{2} \chi^{2}}\right. \\
& \left.+S \mathcal{D}_{n}^{(2)}\left(\psi_{0}\right) \nu_{3}\left(1-t_{3}\right)\left[\left(1+t_{3}\right) z_{2}-\nu_{2}\left(1-t_{1} t_{2}\right)\right]\right\}+\widetilde{\mathcal{G}}_{n}\left(-\nu_{2}\right),
\end{aligned}
$$

where

$$
\begin{aligned}
& \mathcal{D}_{n}^{(1)}(y)=J_{\frac{n-1}{2}}(y)-i J_{\frac{n+1}{2}}(y) \\
& \mathcal{D}_{n}^{(2)}(y)=\left(1+i \frac{n-1}{4 y}\right)^{J_{\frac{n-1}{2}}}(y)-i\left(1-i \frac{n+1}{4 y}\right) J_{\frac{n+1}{2}}(y) \\
& \widetilde{\mathcal{G}}_{n}(\nu)=\nu \frac{i^{\frac{n+1}{2}} m e^{3} \chi}{2 \sqrt{2} \pi^{2}} \int_{0}^{\infty} d s \int_{0}^{1} d u[1-2 u(1-u)] \mathrm{e}^{-i\left(s+\psi_{1}\right)} \mathcal{D}_{n}^{(1)}\left(\psi_{1}\right)
\end{aligned}
$$

with $J_{n}(y)$ being the ordinary Bessel functions [40] and where

$$
\begin{aligned}
& z_{1}=2 x_{1}\left(x_{2} \nu_{2}+x_{3} \nu_{3}\right)+2 x_{2} x_{3}-1, \quad z_{2}=2 x_{1}\left(-x_{2} \nu_{2}+x_{3} \nu_{3}\right)-2 x_{2} x_{3}+\nu_{2}, \\
& z_{3}=2 x_{1}\left(x_{2} \nu_{2}-x_{3} \nu_{3}\right)-2 x_{2} x_{3}+\nu_{3}, \quad x_{j}=\frac{s_{j}}{S}, \quad t_{j}=1-2 x_{j}, \\
& \psi_{0}=\frac{4}{3} \chi^{2} S^{3}\left[x_{2}^{2} x_{3}^{2}+2 x_{1} x_{2} x_{3}\left(\nu_{2} x_{2}+\nu_{3} x_{3}\right)+x_{1}^{2}\left(\nu_{2} x_{2}-\nu_{3} x_{3}\right)^{2}\right], \\
& \psi_{1}=\frac{4}{3} \nu^{2} \chi^{2} u^{2}(1-u)^{2} s^{3}, \quad S=s_{1}+s_{2}+s_{3} .
\end{aligned}
$$

The amplitudes $M_{n, 221}$ are also different from zero and they are obtained from $M_{n, 212}$ by the replacement $\nu_{2} \leftrightarrow \nu_{3}$, where $\nu_{2,3}=\left(k k_{2,3}\right) /\left(k k_{1}\right)$, with $k^{\mu}=(\omega, 0,0, \omega)$ being the fourmomentum of the laser photons. We note that the above expressions hold for arbitrary values of the parameter $\chi$ and exhibit a nonperturbative dependence on the laser parameters. 
As we have mentioned, in the case of a circularly polarized laser field, only one or three laser photons can be absorbed from the laser field. The expressions of the corresponding amplitudes are given in [37]. Starting from Eq. (7) one can calculate the differential rate $d \dot{W}_{n, i j k}$ of photon splitting events of a photon with polarization $i$ into two photons with polarizations $j$ and $k$ with the absorption of $n$ laser photons as

$$
d \dot{W}_{n, i j k}=\frac{2 \pi}{\omega_{1}}\left|M_{n, i j k}\right|^{2} d \nu_{2} d \phi
$$

where $\phi$ is the angle between the vector $\boldsymbol{k}_{2 \perp}=-\boldsymbol{k}_{3 \perp}$ and the $x$ axis. In these formulas the perpendicular direction refers to the components of vectors in the plane perpendicular to the laser propagation direction, i.e. the $z$-direction. We now shortly discuss the possibility of an experimental observation of photon splitting in a laser field. Photon splitting in a strong atomic field has already been observed [6]. We consider here the same experimental scheme as employed in [6]. In this experiment, the initial photon beam was a tagged photon beam in order to reduce the background generated by other competing processes like, for example, double Compton scattering off the experimental equipment. By using the parameters envisaged at the ELI facility $\left(\omega=1 \mathrm{eV}, I=10^{26} \mathrm{~W} / \mathrm{cm}^{2}\right)$, we obtain the total rate $\dot{W}=\sum_{n, i, j, k} \dot{W}_{n, i j k} \approx 10^{9} \mathrm{~s}^{-1}$. One cannot employ too large a value of the initial photon energy $\omega_{1}$. In fact, the angular separation between the final photons is proportional to $1 / \sqrt{\omega_{1}}$. Moreover, a minimum distance of order of $1 \mathrm{~cm}$ is necessary to detect the two final photons separately in a calorimeter. In the above example, we used $\omega_{1}=50 \mathrm{MeV}$ in such a way that a separation of $1 \mathrm{~cm}$ is obtained roughly after 70 meters. It is interesting to observe that the rate obtained is quite large. However, the final number of photon splitting events is determined by the laser pulse duration (that is much shorter than the photon beam temporal duration) and by the overlapping between the laser and the photon beam. Now, the laser pulse duration expected at ELI is about $10 \mathrm{fs}$ and the laser repetition rate is $1 \mathrm{~Hz}$. Moreover, the typical revolution frequency of the electron bunch in the accelerator used to produce the tagged photons by Compton backscattering is $1 \mathrm{MHz}$. Hence, a typical total flux of tagged photons of $10^{8}$ photons per second [41] corresponds roughly to only 100 photons per laser pulse. If all these photons pass through the laser beam, we obtain about ten events per hour. However, the transverse beam size of the tagged photon beam $\left(\sim 1 \mathrm{~mm}^{2}\right)$ is much larger than that of a focused optical laser $\left(\sim 10 \mu \mathrm{m}^{2}\right)$, so that the reduction due to the overlap between the two beams is very large.

From the foregoing discussion we conclude that the scheme used in [6] is not suitable to observe photon splitting in a laser field. This could also have been expected. In fact, in the case of photon splitting in an atomic field the target is a piece of material (like bismuth, for example) that is always there and all the tagged photons inevitably hit it. On the other hand, in our case the "target" is present in a very small spatio-temporal interval and only a few high-energy photons can hit it. The spatio-temporal dimensions of the laser beam are directly related to the necessity of having a very intense beam and we cannot improve our estimations in this aspect. On the other hand, a tagged photon beam is required as the energies of the two final photons are measured at the same time and the sum of them has to be equal to the initial photon energy (the energy absorbed from the optical laser is of course negligible). Now, the tagging is necessary because the electrons circulating in an accelerator show a relatively large transverse betatron motion that renders the backscattered photon beam non-monochromatic. One possibility to improve this aspect has been already discussed in [42], where the electrons coming from the undulator of an x-free electron laser can be used to produce almost monochromatic high-energy initial photons, without the necessity of tagging. In this way, photon fluxes up to $10^{11}$ photons per second are envisaged, leading in the above example to $10^{4}$ photon splitting events per hour. Another improvement implicit in this scheme is the much smaller photon beam dimensions than when an electron beam coming from an accelerator is employed.

\section{Conclusions}

In this paper we have reviewed a few processes involving strong laser beams and their interaction in vacuum due to the polarization of vacuum itself. We have seen that by employing strong 
laser beams with power already available, the elusive process of photon-photon scattering can eventually be measured, at least in principle. The importance of taking into account the diffractive effects in the interaction between a probe beam and a strong optical beam has also been pointed out. These effects are neglected in the refractive-index approach usually employed but they have been shown to be quantitatively and qualitatively relevant. Moreover, we have stressed how the peculiar properties of a plasma near the plasma frequency lead to an enhancement of the effects of vacuum polarization. Finally, the process of photon splitting in a laser field has been considered in more detail. This is a very interesting process, offering the possibility of testing QED in the presence of a strong laser field in a nonperturbative regime. However, the photon sources available today are not suitable because they provide photon beams with low fluxes and with dimensions much larger than those of a strong focused laser beam. However, we have seen how new sources of monochromatic high-energy photons could be used in the future to observe photon splitting in a laser field.

\section{References}

1. V.B. Berestetskii, E.M. Lifshitz, L.P. Pitaevskii, Quantum Electrodynamics, 2nd edn. (Elsevier, Oxford, 1982)

2. W. Greiner, B. Müller, J. Rafelski, Quantum Electrodynamics of Strong Fields: With an Introduction into Modern Relativistic Quantum Mechanics (Springer, Berlin, 1985)

3. R.N. Lee, A.I. Milstein, V.M. Strakhovenko, Sov. Phys. JETP 85, 1049 (1997); Phys. Rev. A 57, 2325 (1998); Phys. Rev. A 58, 1757 (1998)

4. R.N. Lee et al., Phys. Rep. 373, 213 (2003)

5. Sh. Zh. Akhmadaliev et al., Phys. Rev. C 58, 2844 (1998)

6. Sh. Zh. Akhmadaliev et al., Phys. Rev. Lett. 89, 061802 (2002)

7. E.S. Fradkin, D.M. Gitman, S.M. Shvartsman, Quantum Electrodynamics with Unstable Vacuum (Springer-Verlag, Berlin, 1991)

8. W. Dittrich, M. Reuter, Effective Lagrangians in Quantum Electrodynamics (Springer-Verlag, Berlin, 1985)

9. W. Dittrich, H. Gies, Probing the Quantum Vacuum: Perturbative Effective Action Approach in Quantum Electrodynamics and its Application (Springer-Verlag, Berlin, 2000)

10. S.L. Adler et al., Phys. Rev. Lett. 25, 1061 (1970)

11. Z. Bialynicka-Birula, I. Bialynicki-Birula, Phys. Rev. D 2, 2341 (1970)

12. S.L. Adler, Ann. Phys. (N.Y.) 67, 599 (1971)

13. C. Rischel et al., Nature 390, 490 (1997); Ph. Zeitoun et al., Nature 431, 426 (2004); A.L. Cavalieri et al., Phys. Rev. Lett. 94, 114801 (2005); J. Seres et al., Nature 433, 596 (2004)

14. G. Mourou, D. Umstadter, Sci. Am. 286, 80 (1997); V. Malka et al., Science 298, 1596 (2002); Th. Katsouleas, Nature 431, 515 (2004); P.D. Mangles et al., Nature 431, 535 (2004); C.G.R. Geddes et al., Nature 431, 538 (2004); J. Faure et al., Nature 431, 541 (2004); J. Fuchs et al., Nature Phys. 2, 48 (2006); B.M. Hegelich et al., Nature 439, 441 (2006); H. Schwoerer et al., Nature 439, 445 (2006)

15. T. Tajima, G. Mourou, Phys. Rev. ST Accel. Beams 5, 031301 (2002); S. Gordienko, A. Pukhov, O. Shorokhov, T. Baeva, Phys. Rev. Lett. 94, 103903 (2005)

16. S.-W. Bahk et al., Opt. Lett. 29, 2837 (2004)

17. J. Schwinger, Phys. Rev. 82, 664 (1951)

18. H. Euler, Ann. Physik 26, 398 (1936); A.I. Ackiezer, Phys. Z. 11, 263 (1937); R. Karplus, M. Neuman, Phys. Rev. 83, 776 (1951)

19. E.B. Aleksandrov, A.A. Ansel'm, A.N. Moskalev, Sov. Phys. JETP 62, 680 (1986)

20. I. Affleck, L. Kruglyak, Phys. Rev. Lett. 59, 1065 (1987)

21. N.N. Rozanov, Sov. Phys. JETP 76, 991 (1993); Sov. Phys. JETP 86, 284 (1998);

22. D. Bernard et al., Eur. Phys. J. D 10, 141 (2000)

23. A. Di Piazza, K.Z. Hatsagortsyan, C.H. Keitel, Phys. Rev. D 72, 085005 (2005)

24. E. Lundström et al., Phys. Rev. Lett. 96, 083602 (2006)

25. A. Di Piazza, K.Z. Hatsagortsyan, C.H. Keitel, Phys. Rev. Lett. 97, 083603 (2006)

26. T. Heinzl et al., Opt. Commun. 267, 318 (2006)

27. Y.I. Salamin et al., Phys. Rep. 427, 41 (2006)

28. G.A. Mourou, T. Tajima, S.V. Bulanov, Rev. Mod. Phys. 78, 309 (2006) 
29. M. Marklund, P.K. Shukla, Rev. Mod. Phys. 78, 591 (2006)

30. D.L. Burke et al., Phys. Rev. Lett. 79, 1626 (2006)

31. V.O. Papanyan, V.I. Ritus, Sov. Phys. JETP 34, 1195 (1972); Sov. Phys. JETP 38, 879 (1974)

32. P.B. Corkum, Phys. Rev. Lett. 71, 1994 (1993)

33. ELI project homepage at http://www.extreme-light-infrastructure.eu

34. E.E. Alp, W. Sturhahn, T.S. Toellner, Hyperfine Interact. 125, 45 (2000)

35. A. Di Piazza, K.Z. Hatsagortsyan, C.H. Keitel, Phys. Plasmas 14, 032102 (2007)

36. A.I. Akhiezer, R.V. Polovin, Sov. Phys. JETP 3, 696 (1956)

37. A. Di Piazza, A.I. Milstein, C.H. Keitel, Phys. Rev. A 76, 032103 (2007)

38. V.N. Baier, V.M. Katkov, A.I. Milstein, V.M. Strakhovenko, Sov. Phys. JETP 42, 400 (1975)

39. V.N. Baier, A.I. Milstein, V.M. Strakhovenko, Sov. Phys. JETP 42, 961 (1976)

40. M. Abramowitz, I. Stegun (eds.), Handbook of Mathematical Functions (Dover Publications, New York, 1972)

41. S.J. Hall, G.J. Miller, R. Beck, P. Jennewein, Nucl. Instrum. Meth. A 368, 698 (1996)

42. V.N. Litvinenko et al., Phys. Rev. Lett. 78, 4569 (1997) 